\title{
Le déplacement : une dimension d'analyse et une modalité pour comprendre les relations entre nature, science et société
}

Displacements: an Analysis Dimension and a Methodology to understand the Relationships between Nature, Science and Society

Igor Babou

\section{CpenEdition Journals}

Édition électronique

URL : http://journals.openedition.org/questionsdecommunication/2695

DOI : 10.4000/questionsdecommunication.2695

ISSN : 2259-8901

\section{Éditeur}

Presses universitaires de Lorraine

\section{Édition imprimée}

Date de publication : 30 juin 2011

Pagination : 215-234

ISBN : 978-2-8143-0084-2

ISSN : 1633-5961

\section{Référence électronique}

Igor Babou, «Le déplacement : une dimension d'analyse et une modalité pour comprendre les relations entre nature, science et société », Questions de communication [En ligne], 19 | 2011, mis en ligne le 21 juin 2018, consulté le 10 décembre 2020. URL : http://journals.openedition.org/

questionsdecommunication/2695; DOI : https://doi.org/10.4000/questionsdecommunication.2695 


\section{$>$ NOTES DE RECHERCHE}

IGOR BABOU

Centre Norbert Elias, Marseille

École normale supérieure, Lyon

igor.babou@orange.fr

\section{LE DÉPLACEMENT : UNE DIMENSION D'ANALYSE ET UNE MODALITÉ POUR COMPRENDRE LES RELATIONS ENTRE NATURE, SCIENCE ET SOCIÉTÉ}

Résumé. - L'objet de cet article est la notion de « déplacement » et la manière dont elle peut être articulée aux concepts de territoire, de champ, de légitimité et de médiation. II s'appuie, d'une part, sur une lecture des utilisations du déplacement comme analyseur ou comme méthode dans la littérature sociologique et communicationnelle, d'autre part, sur un ensemble de recherches empiriques portant sur les relations entre sciences et société, ainsi que sur des problématiques environnementales. Le déplacement fournit à l'enquête ethnographique un objet pour l'analyse, mais aussi les modalités de sa mise en œuvre. Par ailleurs, il constitue une dimension de l'analyse communicationnelle des discours. Dans ces deux cas, il a pour enjeu d'unifier une diversité de phénomènes tout en évitant les métaphores textuelles ou narratologiques qui aplatissent l'hétérogénéité de ces phénomènes, en particulier dans la sociologie de la traduction.

Mots clés. - Déplacement, ethnographie, nature, sciences, communication. 
I y a bien longtemps, pour le meilleur comme pour le pire, à travers les récits de voyages, les utopies, les colonisations ou les explorations scientifiques, les

sciences sociales ont construit leurs savoirs dans le contexte de déplacements depuis l'Europe vers des contrées lointaines, réelles ou imaginaires. C'est sans doute à l'anthropologie que l'on doit les derniers feux contemporains de cette vogue du déplacement comme moteur de la connaissance, un récit ethnographique comme Tristes tropiques ayant sans doute constitué le point culminant de cette démarche. Puis est venu le temps où la distance a perdu de sa légitimité au profit d'une anthropologie du proche, et de conceptions moins aventureuses de l'exploration de la planète et des sociétés humaines : on célèbre la fin des frontières, I'hybridation généralisée des sociétés, des genres, des espèces, des territoires, des techniques et des savoirs. Nous vivrions dans un village global, et non plus entourés par l'immensité d'espaces à découvrir en les arpentant. Les sciences sociales se sont également construites en opposition à la nature qu'elles ont souvent conceptualisée comme leur « Autre »'. Cette prise de distance leur a partiellement servi de modèle de rationalité. Du règlement de cette question de la nature dépendent donc certaines modalités de la construction de leurs objets de recherche. Aujourd'hui, cette prise de distance semble poser plus de problème qu'en résoudre tant la nature revient par là où on l'avait chassée du champ des réflexions.

\section{Les sciences sociales confrontées au retour du thème de la nature}

D'une part, la question de la nature réapparaît par la place qu'elle prend politiquement et quotidiennement dans les représentations et les pratiques sociales : par le chemin de la culture, donc. Elle revient, d'autre part, par le biais des catastrophes, des dérèglements, par les enjeux de la rencontre entre l'homme et l'animal dans les territoires où ils entrent en concurrence (Ethnologie française, 2009), bref, par l'imprévisibilité de son action qui contraint les sociétés humaines à la repenser. C'est d'ailleurs l'une des raisons pour laquelle la définition de la nature que donne André Gorz (2008 : 44) est d'un grand intérêt, et qu'elle soustendra les conceptions de cet article :

« À la différence des systèmes industriels, l'écosystème naturel possède une capacité autogénératrice et autoréorganisatrice qui, due à son extrême diversité et complexité, lui permet de s'autoréguler et d'évoluer dans le sens de la complexité et de la diversité croissante. Cette capacité d'autorégénération et d'autoréorganisation est endommagée par des techniques qui tendent à rationaliser et à dominer la nature, à la rendre prévisible et calculable ».

\footnotetext{
' Pour la période contemporaine, le symptôme de l'opposition des sciences sociales à la nature apparaît à travers l'histoire de la difficile et tardive prise en compte de l'environnement par la sociologie (Boudes, 2008). Cependant, il reste qu'on ne peut résumer ni la « modernité », ni l'ensemble des sciences sociales, à une épistémè uniforme qui serait caractérisée par une défiance vis-à-vis de la nature: on ne pointe ici qu'une tendance.
} 
Outre l'accent que cette définition met sur le caractère autopoiétique de la nature, elle a l'avantage de ne pas en exclure l'homme (qui possède, comme chaque espèce naturelle, la capacité d'autogénération) tout en permettant de poser une différence entre la dynamique naturelle dans son autonomie vis-à-vis de la technique, et les artefacts techno-naturels (par exemple les organismes génétiquement modifiés) dont l'origine ne peut se comprendre sans une action industrielle et scientifique préalable. De même, ce critère de l'autopoïèse de la nature permet de penser l'action humaine sur la nature comme ayant transformé cette dernière dans le cours historique, tout en reconnaissant au « fait » naturel une spécificité : la capacité d'autopoïèse serait ce qui résiste à toute définition constructiviste de la nature. Les travaux de Philippe Descola (2002, 2005) renforcent l'intérêt de ce type de conception dans la mesure où l'on ne peut plus faire comme s'il existait une « essence » de l'espèce humaine qui serait celle d'une domination technicienne et prédatrice de la nature : d'autres capacités à se penser comme faisant partie intégrante de la nature ont existé et existent encore; ce qui montre le caractère ethnocentré des conceptions constructivistes de la nature selon lesquelles cette dernière ne se définirait que par l'intervention et la gestion humaine. S'il est évident qu'il importe de comprendre les processus de la gestion humaine de la nature, cela ne signifie pas pour autant, comme certains géographes se plaisent à le dire quand ils interviennent dans les débats médiatiques à propos d'environnement, que l'idée qu'on pourrait donner des aspects du fonctionnement de la nature indépendamment de l'action humaine ne serait qu'un mythe. L'inventaire de ces interventions de géographes dans le débat public reste à faire. Voici cependant, à titre d'exemple, l'extrait d'un article de Libération (28/09/99) qui rendait compte d'un festival international de géographie ${ }^{2}$ :

«"II n'y a plus de vraies forêts naturelles en France" assure Paul Arnould, biogéographe à l'école normale supérieure de Saint-Cloud. L'idée d'une forêt "primaire", c'est-à-dire naturelle, le "hérisse" : "c'est un mythe, l'image de l'Éden avant que l'homme vienne foutre sa merde". "Pour les géographes, la nature envisagée dans le sens de ce qui n'est pas humanisé n'existe pas. Les traces des sociétés sont présentes partout", tranche Jean-Robert Pitte, président du Festival de géographie, dont la dixième édition est consacrée à la "nature" [...]. Contre les écologistes scientifiques ou politiques, les géographes réhabilitent le mot "exploitation" de la nature : "c'était un terme noble, remarque Paul Arnould. II est devenu négatif avec la pensée marxiste et le système colonial". Ferraillant contre les "khmer verts", Jean-Robert Pitte refuse de considérer I'humanité "comme une espèce simplement prédatrice" [...]. L'un d'entre eux va même jusqu'à dénoncer "l'imposture écologiste" ».

En géographie, l'idée que la nature ne serait qu'une représentation sociale a succédé depuis les années 70 au naturalisme initial de cette discipline avec, cependant, des retours de flammes récents qui interrogent ce mouvement et parfois le contestent ${ }^{3}$. Les sciences humaines et sociales, assimilent souvent la

2 Les positions exprimées dans cet article sont loin d'être des exceptions en géographie.

${ }^{3}$ Sur ces évolutions et les aspects contemporains de cette problématique en géographie (voir Arnould, Glon, dirs, 2005). 
prise en compte de la nature à l'idéologie d'un retour à une origine pure et vierge de toute humanité. Dans sa version politique, l'écologie serait alors l'alliée objective d'idées réactionnaires ${ }^{4}$. Ces caricatures restent marginales, mais la prise en compte de la nature est souvent empreinte d'ambiguités, en particulier quand elle est sous-tendue par l'idée que la nature serait avant tout une construction sociale, une représentation. Elle est rarement considérée comme un phénomène empirique inclus dans les descriptions sociologiques. Les travaux issus de l'école tourainienne vont dans ce sens, dans la mesure où ils abordent la nature principalement à travers les mouvements sociaux $x^{5}$. Dans d'autres secteurs de la socio-anthropologie de l'environnement, l'importance accordée à la thématique de la gestion de la nature correspond au même primat accordé au social ${ }^{6}$. En définissant la nature avant tout comme une représentation, les sciences sociales ne se trouvent-elles pas en contradiction avec l'une des principales théories de la représentation, celle de Charles S. Peirce (1978), pour qui il ne saurait exister de signe indépendant de tout processus référentiel, et chez qui la dimension symbolique de la représentation laisse une large place aux objets du monde? L'expulsion du référent comme dénaturalisation et institutionnalisation du langage chez Ferdinand de Saussure, s'oppose en effet à la conservation du référent, au maintien d'une place pour les signes naturels chez Charles S. Peirce. Ce qui distingue fondamentalement Ferdinand de Saussure de Charles S. Peirce et qui, au-delà, le distingue de tous ceux qui l'ont précédé, c'est le choix en faveur d'un modèle binaire, et non ternaire, de la signification. Le linguiste suisse s'inscrit en rupture par rapport à la philosophie du langage qui conceptualisait le sens des mots de manière ternaire: depuis Aristote et jusqu'aux grammairiens de Port Royal, les choses étaient reliées aux concepts que l'esprit s'en formait par l'intermédiaire des mots du langage ${ }^{7}$. La sémiologie saussurienne, telle que nous l'a léguée le Cours de linguistique générale (1916), déplace le langage à bonne distance de la nature, en éliminant les choses du monde, alors que la sémiotique de Charles S. Peirce, parce qu'elle conserve le référent et la structure ternaire de la signification, inscrit les processus de signification dans la naturalité, sans pour autant négliger leur dimension sociale. Cette opposition épistémologique, au sein des théories de la représentation et du langage, est-elle pour autant évoquée et tranchée lorsque le choix est fait de penser la nature sous forme de « représentation », ou de « construction sociale »? À ma connaissance non, ce qui ne va pas sans poser des problèmes de cohérence épistémologique.

\footnotetext{
${ }^{4}$ Voir Ferry (1992), dont la critique de l'écologie profonde fondée sur une rhétorique de l'amalgame, ne tient pas compte des engagements pratiques de l'écologie politique.

${ }^{5}$ Vont dans ce sens une grande partie des travaux présentés dans la collection « Sociologies et environnement » des éditions L'Harmattan dirigée par S. Juan, notamment, Actions et enjeux spatiaux en matière d'environnement (2007).

${ }^{6}$ Voir, par exemple, A. Selmi (2006), A. Selmi et V. Hirtzel (2007).

7 Sur ce thème de la triade sémiotique dans l'histoire des théories linguistique, voir Fr. Rastier ( 1990 : 5-39). Concernant Port Royal et l'histoire des théories linguistiques, voir S. Auroux (1996).
} 


\section{La nature et l'enquête de terrain, ou l'engagement du corps contre la bureaucratie scientifique}

À une nature conçue comme dominée et socialement construite, à une wilderness reléguée au rang de lubie romantique, correspondent des conceptions bureaucratiques et souvent immobiles du travail de la recherche en sciences sociales : les conditions d'exercice de notre métier paraissent de moins en moins régies par les nécessités de l'enquête de terrain, et de plus en plus contraintes par celles de l'appartenance à la centralité d'une institution qui exige et contrôle notre présence en son sein. Ce thème d'une bureaucratie scientifique spécialisée, inscrite dans la division du travail et s'opposant au terrain comme lieu vers lequel on se déplace pour rencontrer un espace de confrontation à soi et aux autres, souvent dans un cadre « naturel » (la steppe du Mato Grosso ou les forêts pluviales canadiennes, les vastes plaines américaines ou les déserts du Maghreb, etc.), est ancien et fondateur en ethnologie. Le travail de Vincent Debaene (20 I 0) permet de comprendre à quel point l'ethnologie du début du $X X^{e}$ siècle, celle de Marcel Griaule, Michel Leiris, Alfred Métraux, Jacques Soustelle, puis Claude Lévi-Strauss, a à voir avec un refus de la spécialisation bureaucratique du travail dans les sciences sociales, l'homme d'action qu'était supposé être l'ethnologue s'opposant alors au savant de cabinet, au philosophe et à l'esthète confiné dans sa bibliothèque ou dans son musée. On pourra même faire remonter au XIX siècle cette opposition entre un terrain vers lequel l'ethnologue se déplace, et les obligations bureaucratiques qui lui sont faites par l'université, avec l'exemple de Franz Boas (1909) ${ }^{8}$ qui revient plusieurs fois, dans l'introduction de son livre sur les Indiens Kwakiutl, sur le fait qu'il a du utiliser ses vacances pour mener à bien ses recherches, ou sur le constat que ses charges administratives l'empêchaient de mener correctement son travail de recherche. Reste que l'engagement du corps de l'ethnologue, sa confrontation existentielle au terrain en opposition à la tradition lettrée du savant de cabinet, n'a pas été thématisée par les ethnologues dans leurs écrits académiques, mais plutôt dans les livres de voyages, plus ouverts à la subjectivation du terrain, que la plupart d'entre eux ont écrit en écho à leurs productions académiques (Debaene, 20I0). Ce thème de la confrontation au terrain du corps de l'ethnologue, dans le cadre d'un déplacement s'opposant aux contraintes bureaucratiques d'une profession devenue spécialisée, est donc ancien et lancinant. Cela ne lui retire cependant rien de son intérêt, ni de son actualité.

Dans l'ensemble, à force de se positionner contre ce qu'elles considèrent comme de dangereuses idéologies ou comme des questions trop inquiétantes pour s'inscrire dans les écrits académiques - l'amour pour la nature, le sensible, la subjectivité et le corps dans l'enquête - les sciences sociales ne se retrouventelles pas piégées par cette distance qu'elles ont construite avec la nature et avec les engagements des acteurs? En effet, dans ces domaines, leur position

8 Bien que publié en 1909, le livre rend compte de déplacements effectués entre 1883 et 1897. 
n'a rien de la neutralité axiologique à laquelle elles prétendent. Pour toutes les raisons qui viennent d'être évoquées, la question qui se pose est donc celle de la légitimité théorique d'une conception de la nature uniquement pensée comme un arrière plan, ou vue seulement comme un lieu de projection des actions humaines, c'est-à-dire exclusivement comme une construction sociale ou symbolique. Ce qui ne signifie pas, bien évidemment, qu'il serait illégitime ou sans intérêt d'analyser les mécanismes de la construction sociale et symbolique de l'idée de nature. Enfin, cette question du rapport à la nature dans l'enquête ne semble que très difficilement séparable de celles de la subjectivité et du rapport au corps de l'enquêteur, de même que de prises de positions plus diffuses contre des conceptions bureaucratiques ou philosophiques des sciences sociales.

\section{Intégrer les dynamiques naturelles à la description du social?}

Si l'on ne se contente plus d'une conception de la nature comme lieu de projection des actions humaines, comment intégrer la dynamique des phénomènes naturels à la description du social? J'aimerais proposer ici une démarche à la fois empirique et théorique qui vise, d'une part, à intégrer certaines dynamiques naturelles à la description du changement social et, d'autre part, à unifier la description de phénomènes sociaux et discursifs que l'on rencontre dans le champ de l'analyse des relations entre l'homme et la nature, et dans celui des relations entre sciences et société. En effet, la démarche que je propose a été élaborée dans ces domaines où se rencontrent les enjeux de la matérialité et de l'évolution biologique et sociale. C'est la notion de déplacement que je présenterai comme moyen d'opérer cette unification. Cette notion n'a cependant aucune prétention à épuiser la richesse de ce qui peut être vécu et décrit par les groupes sociaux et leurs observateurs, pas plus qu'elle ne correspond à une abstraction philosophique: son ancrage dans les terrains et corpus qui lui donnent sens reste essentiel. Toute tentative de prise en compte de facteurs naturels par les sciences sociales fait peser sur soi le soupçon d'évolutionnisme ou de soumission à l'impérialisme des sciences de la nature?. À l'exception de la géographie, l'intégration de l'environnement par les sciences sociales est d'ailleurs récente et assez marginale (Boudes, 2008). Toutefois, je pense que l'on peut trouver une position qui ne soit ni aveugle aux déterminants naturels du fonctionnement social, ni sous-tendue par un évolutionnisme biologique. L'opposition radicale entre une conception de l'histoire humaine comme séparée de l'évolution naturelle et une conception de la société comme forme de la nature (Moscovici, 1972) semble aujourd'hui bien forcée. Intégrer la dynamique de la nature à la description du social, oui, mais non sans intégrer également le symbolique. Tenir compte de l'évolution biologique, oui, mais sans en faire le point origine de l'histoire humaine, question

\footnotetext{
9 Pour une critique de l'évolutionnisme, voir S. Juan (2006).
} 
qui - comme celle de l'origine des langues - reste une lubie métaphysique ${ }^{10}$. Considérer les dimensions naturelles ou encore matérielles" non comme des causes finales et uniformes, substrat génétique ou déterminisme technologique, mais comme des composantes de l'ensemble des phénomènes qu'il importe de prendre en compte si l'on veut mieux comprendre l'élaboration des relations entre l'homme et la nature, mais aussi si l'on veut décrire les enjeux pour lesquels les groupes sociaux ou les individus se positionnent vis-à-vis de la nature et des sciences.

Je vais donc présenter la manière dont j'articule l'idée de déplacement à d'autres notions: médiation, territoire, champs, et légitimité. Cette réflexion s'appuie sur un ensemble de publications auxquelles le lecteur pourra se reporter pour le détail des résultats empiriques (Babou, 2004, 2009; Babou, Le Marec, 2003, 2008).

\section{Le déplacement : une notion en émergence}

Dans le domaine de la socio-anthropologie de l'environnement, le déplacement d'espèces animales a été utilisé pour mettre en évidence des phénomènes à l'articulation du naturel et du social, et pour comprendre la manière dont la nature contraint les sociétés humaines à composer avec elle. Par exemple, Marie Roué (2009) montre comment la migration de l'oie bernache du Canada révèle des conflits latents entre peuples autochtones, chasseurs allochtones, biologistes et défenseurs de l'environnement. De mon côté, c'est en m'appuyant sur I'histoire de la migration des baleines franches australes sur la côte argentine que j'ai pu montrer comment un haut lieu touristique de whale watching a pu se constituer, conjointement à plusieurs vagues de migrations humaines depuis les centres urbains vers la Patagonie atlantique (Babou, 2009). De plus, l'étude des migrations des baleines a permis de comprendre certains aspects de la concurrence entre deux équipes de biologistes de la conservation travaillant sur cette zone. Je présenterai plus bas ce travail. Dans ces recherches, le déplacement des oies et des baleines au sein des territoires que ces espèces partagent avec les sociétés humaines est le moteur d'une analyse qui n'est plus uniquement ethnographique, mais qui devient éco-ethnographique. Cela permet d'articuler précisément les savoirs scientifiques et profanes, l'organisation sociale (du travail, du tourisme, des sciences, du débat public politique), et les dispositifs matériels et communicationnels impliqués localement. Véronique Servais (2005), quant à elle, a insisté sur la façon dont les humains interprètent leurs relations avec les animaux sous l'angle de la communication : ainsi le déplacement des dauphins

\footnotetext{
10 Sur la question de l'origine des langues comme thème métaphysique à proscrire des sciences humaines, voir le travail de S. Auroux (2007).

" || me semble que les conceptions évolutionnistes sont très proches des conceptions matérialistes de l'Histoire. C'est en tout cas ce qui apparaît à la lecture de S. Juan (ibid.).
} 
lors des « rencontres enchantées » avec certains groupes d'amateurs qui nagent avec eux est-il interprété comme une désignation, ou une reconnaissance, des humains par les animaux. Ce phénomène induit alors des interprétations en termes de communication interspécifique. On trouve enfin le travail de Florence Weber (1998) qui tient compte des déplacements fréquents de sociologues et de diverses notabilités depuis les centres urbains vers les jardins ouvriers dans la construction de l'imaginaire de ces jardins. Son approche met l'accent sur les légitimités engagées dans ces déplacements entre des territoires qui sont à la fois physiques et symboliques.

Certains historiens ont également pointé le rôle du déplacement dans l'histoire des pratiques de connaissance. Ainsi, dans Lieux de savoir dirigé par Christian Jacob (2007), trouve-t-on deux sections correspondant à ce thème. L'une est consacrée aux itinérances du savoir (par exemple, le lien entre les pratiques de la rhétorique dans l'antiquité grecque ou à l'époque des royaumes combattants de la Chine féodale et la mobilité des enseignants et de leurs disciples). L'autre précise les logiques spatiales du savoir au cours de l'histoire, la géographie de l'internet, etc. II serait trop long de rendre compte de ces approches ici, mais remarquons que la notion de déplacement ne fait pas l'objet d'une élaboration théorique dans cet ouvrage. Du côté de l'histoire de la communication, Armand Mattelart ( 1994) rappelle les origines très matérielles du concept de communication (voies fluviales, ouvrages d'art, routes, etc.), ainsi que les divers modèles de connaissance qui en ont structuré la pensée: organicisme biologique, réseaux, planification industrielle et internationalisation des échanges, mathématisation du social. Là encore, le déplacement, des corps, des informations et des biens, est fondateur. Mais il reste implicite et n'est pas développé comme un concept.

Dans le domaine de l'analyse communicationnelle des discours à propos de sciences, la dimension du déplacementa été mobilisée comme marqueurempirique (Fouquier, Véron, 1985; Babou, 1999, 2004). Par exemple, la représentation du déplacement des journalistes vers les laboratoires de recherche ou, inversement, le déplacement des chercheurs vers les plateaux de télévision, fournissent des indicateurs de l'évolution diachronique de relations de légitimité entre le champ journalistique et le champ scientifique. Décrire qui se déplace vers qui, ou qui est représenté comme se déplaçant vers qui, et selon quelles modalités, donne en effet accès aux légitimités respectives des territoires physiques et symboliques occupés par les acteurs (bureaux, institutions, laboratoires, plateaux de télévision, etc.). Ensuite, c'est dans le domaine de la muséologie des sciences que cette grille d'analyse des discours a été appliquée. Les déplacements (ou l'absence de déplacement) d'objets importés depuis les espaces scientifiques vers les musées de sciences, au cours de leur histoire, fournissent en effet des indices de rapports de légitimité et de conceptions du rapport au savoir et à la vérité (Babou, Le Marec, 2003).

Dans le domaine de la socio-anthropologie des sciences, Bruno Latour (1985), Michel Callon (1986) ou encore Steven Shapin (1991) ont particulièrement 
insisté sur l'importance du déplacement des inscriptions scientifiques pour emporter la conviction des chercheurs et mobiliser des alliés au moment de la constitution des faits scientifiques. Ces déplacements de signes, d'énonciateurs en énonciateurs, expliqueraient mieux que tout esprit scientifique ou que toute méthode rationnelle, la spécificité du travail des scientifiques. Les quelques arguments notés plus haut à propos de Ferdinand de Saussure et de Charles S. Peirce indiquent déjà que je fais plus le constat de l'hétérogénéité des pratiques et de la communication - toujours à la fois matérielles, sociales et symboliques - que celui de leur homogénéité sémiologique. Pierre Bourdieu (200l a :55-60) a sévèrement critiqué ce « textisme » qui aplatit toutes les pratiques et traite la science comme une pratique littéraire qui n'exercerait qu'un simple « effet de vérité ». Certes, dans la description que fait Michel Callon (1986) des tentatives de domestication des coquilles Saint-Jacques dans la baie de Saint-Brieuc, ces dernières résistent à leur enrôlement par les scientifiques quand ils mettent en œuvre des stratégies d'intéressement des acteurs humains et non humains qui constituent leur environnement. Du moins, elles ne se comportent pas exactement comme les scientifiques le prévoyaient. Pour autant, elles n'en sont pas actives, mobiles et ne font aucun choix intentionnel : au mieux, on peut dire qu'elles ne sont pas là où on les attendait, ce qui n'en fait pas des « acteurs » équivalents aux scientifiques ou aux pêcheurs. Qu'elles aient ou non résisté lors de l'étude de Michel Callon, il est difficile de dire si cela aurait changé grandchose à une méthode de description qui consiste à sémiotiser ces mollusques en qualifiant de « porte-paroles » ceux qui sont domestiqués et mobilisés en tant qu'échantillon représentatif dans les argumentations des scientifiques. Ils sont comparés par Michel Callon à des représentants syndicaux en train de « négocier» leur identité avec les acteurs humains, tandis que les crustacés résistants (qui ne répondent pas positivement aux tentatives de domestication) sont comparés à la masse silencieuse d'électeurs ou d'ouvriers non syndiqués et susceptibles de faire basculer à tout moment la situation. L'interprétation peut alors se déployer librement à leur propos, l'« acteur » n'ayant aucun moyen empirique de contester ses interprètes. En termes de description, je ne suis donc pas convaincu du caractère suffisant de la traduction métaphorique, sous forme de textes, des objets matériels ou des entités naturelles mobilisées par la pratique scientifique. Je ne suis pas plus convaincu par leur conversion en actants d'une narration qui rendrait compte à elle seule de l'hétérogénéité des pratiques au sein d'un espace communicationnel unifié et homogène qui irait du cœur du laboratoire à l'espace public élargi des controverses sociotechniques ${ }^{12}$, espace dont on pourrait se dispenser de décrire les normes, les dispositifs et les champs. Ainsi, au sein des organismes de recherche, l'autonomisation de la communication professionnalisée a-t-elle donné la possibilité à certains acteurs d'occuper des

12 Depuis H. Collins etT. Pinch (1979), les « Social studies of knowledge », se sont orientées vers l'analyse des controverses sociotechniques. En France, ces approches sont connues sous les termes de « sociologie des collectifs », de « théorie de l'acteur-réseau » ou de « sociologie de la traduction ». À ce sujet, voir l'article de R. Barbier et J.-Y.Trépos (2007). 
places de médiateurs entre des pôles structuraux. Entre le laboratoire et le public, s'intercalent des communicants, des banques d'images, une presse proche des formats de la presse d'entreprise, ce qui conduit à mettre en scène un imaginaire du déplacement du savoir depuis des centres (le laboratoire) vers l'extérieur (les médias, le public). Durant ce processus, les opérations de production et de reformulation, ou encore les importations et les conflits de normes qui caractérisent ces médiations, semblent disparaître aux yeux des acteurs : tout se passe comme s'il n'y avait que «transmission d'informations », là où l'analyse empirique révèle des processus de production (Babou, Le Marec, 2008). Si des signes se déplacent pour contribuer à la construction des faits scientifiques, ce phénomène est bien plus hétérogène que l'image qu'en donne la sociologie de la traduction.

En fin de compte, c'est du côté de la sociologie urbaine qu'il faut se tourner pour trouver des élaborations théoriques et méthodologiques proches des nôtres et qui revendiquent des approches sensibles de la connaissance, par exemple à travers le parcours d'un territoire ou d'un quartier par les chercheurs, l'enjeu étant la réintroduction du corps dans le processus de connaissance. À partir du travail fondateur de Jean-François Augoyard (1979) qui a été récemment réédité, les chercheurs du laboratoire Cresson ${ }^{13}$ de Grenoble ont construit le déplacement, et plus spécifiquement la marche à pied dans l'espace urbain, à la fois comme un objet de recherche à part entière et comme une méthode d'investigation. Le déplacement est alors relié aux subjectivités et aux capacités de remémoration de l'observateur et de l'observé (Petiteau, 2008), aux variétés des formes de la marche à pied et aux descriptions qui en rendent compte (Thibaud, 2008), ou à une typologie des passages depuis l'espace privé vers l'espace public, avec une attention accordée aux objets communicants, comme le baladeur audio, qui sont engagés dans ces franchissements d'espaces hétérogènes (Thibaud, 1994). Les revendications d'approches sensibles du terrain ne sont, j'en suis persuadé, pleinement compréhensibles que pour ceux qui mènent euxmêmes des enquêtes de terrain, la pratique de l'enquête risquant toujours d'être dénoncée comme un « mythe scientifique » par ceux qui se sentent plus en phase avec le commentaire érudit de textes savants qu'avec le déplacement en dehors des bureaux et des bibliothèques ${ }^{14}$. Les enjeux du terrain ont pu recevoir diverses formulations selon les disciplines, mais c'est sans doute en sciences de l'information et de la communication, avec le travail de Joëlle Le Marec (2002a : 19), que leur explicitation se fonde le plus radicalement sur l'analyse de la relation communicationnelle à l'Autre, toujours accompagnée de la réflexivité du retour sur soi et d'une conception collective de la recherche qui étend l'idée d'un « espace mental de l'enquête » (Passeron, 1995) à la compréhension

${ }_{13}$ Centre de recherche sur l'espace sonore et l'environnement urbain.

${ }^{14}$ Voir à ce sujet la controverse, dans le champ des recherches en communication, entre Ph. Hert (2005) et S. Olivesi (2005). Sur ce thème du rapport au terrain, je suis également largement redevable aux nombreuses discussions que nous avons dans notre laboratoire avec J. Le Marec, ainsi qu'à son mémoire d'habilitation à diriger des recherches (2002a). 
intersubjective du terrain comme pratique communicationnelle plus que comme pôle d'extériorité où aller chercher des « données ». En effet, pour Joëlle Le Marec (ibid.), il faut

\begin{abstract}
« développer un empirisme malgré (ou avec) la conscience critique qui interdit de croire en la possibilité de séparer des choses et des médiations par lesquelles ces choses se manifesteraient, et qui oblige ainsi à rester dans l'inconfort de catégories floues, émergeant localement des situations de recherche, le point fixe devenant soi-même, manifesté par une attitude réflexive distante des mutations générées par l'action. Mais cet empirisme prétend malgré tout préserver la nécessaire "insouciance" du dynamisme de l'action, sans lequel on ne ferait rien d'autre que de se regarder penser, alors que l'Autre, le pôle d'extériorité, existe bel et bien, évidemment ».
\end{abstract}

Dans ce contexte, le déplacement sur le terrain relèverait finalement d'une relation sociale, avant tout communicationnelle. Ce que l'on peut dire à la suite de cette brève lecture des enjeux du déplacement dans divers champs, c'est qu'il ne s'agit pas d'un concept parfaitement balisé ni largement mobilisé. Ancré dans les terrains et corpus de l'enquête, ce dernier reste implicite, apparaît dans des textes dispersés, et à ma connaissance on ne trouve aucun colloque ni ouvrage ayant tenté de faire le point à son sujet. Son pouvoir heuristique est cependant important et on peut faire appel à lui pour articuler, au plan théorique et empirique, des relations de légitimité à des territoires physiques ou symboliques, à des normes et à des relations entre acteurs et discours sociaux: je développerai cette articulation plus loin. C'est également la matérialité des processus de communication qui peut être interrogée dans différents contextes en s'appuyant sur ce type de conceptualisation. Par son caractère assez général, et par les développements empiriques qu'elle permet, la notion de déplacement paraît donc utile pour travailler le domaine de l'analyse des relations entre nature, sciences et société.

\title{
Déplacement et changement social
}

Je vais prendre le cas d'un terrain ethnographique réalisé en 2008 dans un village situé sur la Péninsule Valdés, en Patagonie argentine. Le terrain portait sur trois champs : le whale watching (l'écotourisme), la biologie de la conservation - très active dans la région - ainsi que sur le débat public à propos d'environnement (Babou, 2009). J'ai été amené à reconstituer les phases de peuplement, puis de repeuplement de ce village par des néo-ruraux, à partir de l'étude d'archives ainsi que d'entretiens réalisés avec des habitants. La lecture d'articles de biologie marine rédigés ou conseillés par les biologistes avec qui je collaborais, m'a permis par ailleurs de décrire diachroniquement les déplacements des baleines franches australes qui ont fait la réputation de ce site touristique: elles arrivent par milliers chaque année à la même date, et y séjournent entre 5 et 7 mois durant leur période d'accouplement, d'accouchement et d'allaitement des baleineaux. Outre ce déplacement annuel, un déplacement plus intéressant est constitué par celui des « nurseries », c'est-à-dire des lieux où les baleines allaitent leurs petits. 
Suite à un problème environnemental d'origine anthropique (les déchets de la surpêche et les déchetteries à ciel ouvert), et à l'augmentation des populations de goélands, il s'est produit un étonnant changement de comportement alimentaire chez ces oiseaux. Ces derniers « attaquent» les baleines et se nourrissent de la graisse et de la couche sous-cutanée du dos des baleines: fait exceptionnel qui ne s'observe qu'à cet endroit de la planète. Pour fuir ces attaques, les baleines ont quitté le golfe du nord qu'elles occupaient avant les années 70 pour se diriger d'abord vers l'est de la Péninsule, puis occuper à partir des années 80 le golfe situé au sud (Rowntree et al., 1998; Rowntree, Payne, Schell, 200I). Or, les seules installations portuaires exploitables pour le whale watching sont justement situées dans le golfe du sud, tandis que les lois de protection environnementale interdisent tout usage touristique du golfe situé au nord. Sur la base de la topographie de ce territoire, la mise en parallèle des deux diachronies de déplacements (d'abord celle des baleines, puis celle des néo-ruraux venus repeupler ce village) permet de comprendre comment l'écotourisme a pu émerger à partir des années 80 et se développer alors que ce même village était ruiné et pratiquement vidé de ses habitants (des gauchos pratiquant l'élevage de moutons) à l'arrivée des premiers néo-ruraux. Par ailleurs, plusieurs équipes de biologie de la conservation sont en concurrence sur place, où elles dépendent, pour la production de leurs données, de l'accès aux baleines et de la localisation d'une infrastructure logistique sur la péninsule. L'installation de l'unique infrastructure logistique de recherche dans le golfe du nord, dans les années 70, est devenue problématique depuis le déplacement des baleines vers le sud, déplacement qui a été exploité tactiquement par une autre équipe de biologistes pour problématiser différemment les questions de conservation ( $y$ compris au plan politique et médiatique). Une équipe a priori moins reconnue a alors pu s'implanter en exploitant habilement sa capacité à être mobile, tandis que l'équipe la plus ancienne se retrouve quelque peu hors champ du fait de sa localisation au nord.

Ces déplacements conjoints ont donc contribué à la construction sociale et historique, mais aussi naturelle, du territoire. Ils ont été des facteurs de changement dans la répartition des ressources économiques, des légitimités scientifiques, ou encore des usages sociaux du territoire et des justifications politiques et médiatiques des stratégies de développement. Sans cette prise en compte de données biologiques, aucune « logique sociale » n'aurait permis de décrire correctement, à elle seule, le peuplement de cette zone et les diverses évolutions des stratégies des acteurs. Une question qui se pose est de savoir s'il faut ne décrire que des déplacements. Évidemment non, ne serait-ce que parce que la description du changement n'a de sens que par la prise en compte de la stabilité. Ceci se traduit par le fait que les déplacements interviennent dans des territoires géophysiques qui évoluent selon des temporalités bien plus lentes que celles des populations humaines ou des espèces animales. Ou encore parce que je ne pouvais pas séparer l'impact symbolique et économique de l'arrivée des flux de populations liées au tourisme, de leur confrontation à des populations 
plus anciennes déjà installées sur place et y ayant développé une culture et des représentations spécifiques de leur rapport à la nature et au territoire. Autrement dit, qu'il s'agisse du déplacement des baleines, de la succession de vagues de peuplement humain, ou encore de la concurrence entre équipes scientifiques, c'est par l'inscription dans un territoire stable, déjà marqué symboliquement et culturellement et servant de support géophysique, que les déplacements font sens en créant du changement ou en étant interprétés comme des facteurs de changement.

\section{Les distances qui construisent la relation entre l'homme et la nature}

Décrire des déplacements est complémentaire d'un autre type d'analyse, celle d'une distance parcourue. Ma proposition consiste à décrire une distance, physique mais également symbolique, contribuant, d'une part, au réglage des relations entre l'homme et la nature et, d'autre part, au réglage des relations entre les pôles structurels - réels ou supposés - d'une organisation sociale ou discursive. II s'agit d'observer des médiations : éléments s'intercalant - matériellement ou symboliquement - entre des pôles qu'ils contribuent dans le même temps à légitimer et à construire en tant que principe d'opposition, comme éléments complémentaires ou comme phases temporelles d'un processus. Pour que des déplacements fassent sens pour les acteurs comme pour l'observateur et pour que la description soit heuristique, on doit être attentif à des déplacements opérant entre des territoires symboliquement marqués, de manière à ce que le franchissement de certains seuils implique une confrontation de légitimités. Les observations doivent donc principalement concerner des relations entre des espaces, physiques ou symboliques, sur lesquels un pouvoir ou une conformité à certaines normes s'exerce, et dont l'éloignement (ou le rapprochement) met en jeu l'identité du territoire et de l'acteur social en déplacement qui en est le représentant. Ainsi aller d'une ville à un espace naturel patrimonialisé nécessite de franchir des espaces géographiques et d'emprunter des réseaux de transports, des infrastructures, éventuellement de se déplacer à pied dans les zones protégées, ou encore d'utiliser les services maritimes d'entreprises de visites touristiques, c'est-à-dire d'articuler entre eux plusieurs modes de transport. Le déplacement permet alors d'éprouver l'articulation et la matérialité de ces médiations : c'est même le déplacement, sa planification et sa mise en œuvre par des stratégies de développement territorial ou par des usages sociaux, qui les articule. Le déplacement permet également de parcourir, de lire, les inscriptions institutionnellesquiénoncentleterritoire.Carlescaractéristiquesd'un déplacement sont également construites, au plan symbolique, par des institutions de gestion et de légitimation du patrimoine naturel (Patrimoine mondial de l'Unesco, parcs nationaux ou régionaux, collectivités territoriales, etc.), par des organismes de normalisation (l'Union internationale pour la conservation de la nature élabore 
depuis 1948 des normes de classement des espaces naturels), par des médias et un champ éditorial (guides de voyage, communication des opérateurs de tourisme, presse « nature » ou écologiste, communication des institutions de tutelle du patrimoine naturel, etc.), ainsi que par des représentations sociales partagées au sein des divers champs de la pratique touristique, scientifique ou encore politique. Dans le contexte de l'espace naturel qui constitue le but d'un déplacement, les médiations et leurs modes d'énonciation peuvent varier et se coordonner : panneaux indiquant les limites du parc et réalisant ainsi à la fois un énoncé performatif de sa sanctuarisation et l'énonciation de l'institution qui le gère, brochures d'information distribuées à l'entrée et précisant les règles d'usage de l'espace naturel, instauration d'un rapport économique (gratuité ou non des espaces naturels), guide assurant la visite en groupe ou encore parcours libre et en autonomie pour les visiteurs. Au cours de ces déplacements, les acteurs ne franchissent pas des « épreuves » : je prends à nouveau ici mes distances avec la métaphore narratologique mobilisée par la sociologie de Bruno Latour et Michel Callon qui donne l'illusion de l'homogénéité des processus. Ils empruntent plutôt des dispositifs et des médiations qui se caractérisent par leur hétérogénéité. En décrivant les déplacements et les médiations qui construisent et règlent la relation entre l'homme et la nature, on évite d'uniformiser métaphoriquement les pratiques tout en se dotant de concepts assez généraux pour analyser de manière cohérente les phénomènes rencontrés.

\section{L'expérience du déplacement par le chercheur}

Pourquoi considérer les déplacements comme des données n'engageant que les acteurs ou les discours que nous décrivons, tandis que l'observateur ne serait pas concerné? Le fait de constituer un corpus implique souvent le déplacement vers un centre d'archives, de même qu'une enquête de terrain impose un déplacement vers un lieu proche ou lointain. Ces déplacements font partie des conditions de possibilité de la connaissance. Ils s'accompagnent de contacts et de communications (avec les acteurs, avec les documentalistes, etc.) qui ont des répercussions sur les connaissances produites. Les recherches de Joëlle Le Marec (2002b) ont amplement développé l'idée que le terrain sociologique constituait non pas un lieu de recueil de données extérieures à la situation d'enquête, mais que cette dernière était une situation de communication. Je ne développerai donc pas ici cet aspect, le déplacement du chercheur vers les acteurs ou vers ses corpus étant l'une des composantes de cette situation de communication vécue dans la pratique de recherche. Pour ma part, ce que j'ai pu expérimenter est l'enjeu du parcours d'un territoire pour la compréhension du rapport à la nature. Je décrirai ici cet enjeu sous deux modalités correspondant à deux situations vécues sur le terrain, à nouveau en Argentine. 
La première a consisté à me retrouver transporté en voiture par un informateur pour une visite de son village et de son environnement immédiat. Le parcours réalisé traduisait partiellement le regard de l'informateur sur le territoire. Dans le cas de cet informateur qui refusait tout entretien enregistré et déclarait qu'il n'était pas intéressé par les baleines et leurs problèmes (qui étaient l'objet de mon enquête), cela m'a permis de comprendre les catégories à partir desquelles se construisait son rapport à la nature : des critères d'appréhension de l'espace et de l'environnement qui ne m'étaient pas apparus auparavant, et dont les biologistes avec qui je collaborais ne tenaient pas compte. Alors que l'attention des habitants étaient focalisée sur le problème des baleines, lui s'attardait sur le problème des décharges situées à l'orée du village, sur l'évacuation mal gérée des eaux usées ${ }^{15}$, ou encore sur l'érosion des dunes et les risques de débordement des cañons en cas de fortes pluies : là où les biologistes voyaient la nature (c'està-dire, pour eux, les baleines), lui voyait de l'économie, tandis que là où il voyait des problèmes environnementaux, les biologistes ne voyaient rien d'utile à leur problématique. Quant à moi, je n'avais pas perçu ces problèmes, en particulier celui des cañons, faute d'avoir assisté à une forte pluie. II a fallu pour cela qu'il me fasse voir avec ses yeux « son » territoire, et que je prenne conscience, à chaque arrêt du véhicule, des lieux et des phénomènes mobilisant son intérêt là où nos précédentes discussions n'avaient pas été d'une grande utilité.

Une autre situation consiste à se déplacer, en variant les moyens de transport, pour mettre à l'épreuve ses propres échelles d'observation. II s'agit de vivre les temporalités et les étapes d'un parcours, de manière à percevoir, en l'expérimentant sur soi, la signification de l'espace, du territoire ou de l'environnement naturel telle qu'elle peut être construite par les acteurs locaux. C'est à ce type d'arpentage d'un territoire que je me suis longuement livré à un moment où je commençais à adhérer trop fortement aux regards de mes informateurs sur ce même territoire. C'est en m'imposant des changements d'échelles, en réalisant des sorties hors du village où je résidais, de manière à avoir une vision extérieure de ce village depuis le désert qui l'entourait, que j'ai pu tout à la fois relativiser l'écœurement partagé avec les acteurs locaux pour les dégradations environnementales qu'ils ressentaient fortement dans leur village. tout en ne disqualifiant pas ces mêmes perceptions au nom d'une rationalité abstraite et surplombante, quand je revenais au village depuis le désert. Par rapport à l'immensité du territoire ou à la pollution générée par la ville industrielle située de l'autre côté du golfe, les dégradations du village paraissaient en effet mineures. Les allers et retours successifs du village vers le désert ou vers la ville, m'ont permis de me mettre littéralement - et surtout physiquement - à la place de gens vivant quotidiennement dans ce lieu et qui n'en sortaient guère : en sortir permettait de prendre mes distances avec mon adhésion avec le sentiment général, tout en comprenant comment ce problème, par sa proximité, devenait

15 L'informateur était employé à l'usine de désalinisation de l'eau de mer qui fournissait le village en eau potable. 
un cadre de pensée aussi envahissant dans les conversations quotidiennes. Entre la prise de conscience de cette distorsion psychologique induite par la trop longue fréquentation du « dedans » d'un lieu et du point de vue des acteurs, et la compréhension théorique que j'aurais pu en avoir si je l'avais lue dans un article scientifique - comme le lecteur qui découvre ce texte en me lisant aujourd'hui -, il y a l'importante marge entre la compréhension physique d'un phénomène, et une compréhension littérale, ou théorique. Non pas que l'une soit supérieure à l'autre, mais la compréhension physique a, dans le cas du rapport à la nature, un caractère de nécessité épistémologique à partir du moment où l'on ne se satisfait plus de la seule conception de la nature comme « représentation » ou comme « construction sociale ». Travailler sur le rapport entre l'homme et la nature revient à éprouver ce que la nature fait à l'enquêteur, du vent glacial descendu des Andes à la monotonie d'un paysage, du souffle des baleines aux mugissements des lions de mer, de l'odeur subtile de la marée à la puanteur des décharges à ciel ouvert, etc. Éprouver et incorporer certaines dimensions du rapport à la nature enrichit considérablement une compréhension théorique liée à des lectures ou à l'analyse du verbatim d'entretiens de gens parlant de leur rapport à la nature, car c'est aussi de perception sensorielle qu'il s'agit, et la perception ne peut être textualisée sans perdre beaucoup ce qui en elle fait sens. Contrairement à ce que le rationalisme prétend, c'est par l'engagement direct du corps, de la perception et des émotions, qu'il est possible - et dans le cas du rapport à l'environnement naturel, nécessaire - de produire de la connaissance, et qui plus est une connaissance empreinte de réflexivité.

\section{Conclusion}

Tout déplacement est potentiellement porteur ou créateur de sens, qu'il s'agisse du déplacement d'un objet matériel, de celui d'un animal ou d'une espèce, d'une personne ou d'un groupe, ou encore d'un signe ou d'un ensemble de signes. Potentiellement car, pour que du sens soit créé, encore faut-il quelqu'un pour l'expérimenter, le produire ou l'interpréter: en cela, le déplacement s'inscrit dans le même type de phénoménologie que l'indice. Sans observateur, un déplacement porte un sens potentiel, mais n'en crée pas. Tout déplacement relie, mais aussi crée en les désignant à l'attention des phénomènes qui paraîtront alors s'inscrire soit dans une structure d'opposition, soit dans des complémentarités, soit encore faire partie des étapes d'un processus plus complexe. Ce faisant, les déplacements permettent de mesurer des distances, physiques ou symboliques, qui sont ainsi désignées au sein de structures, de dispositifs, de territoires, de champs, de discours, d'images, etc. C'est-à-dire au sein de phénomènes pouvant être aussi bien matériels que sociaux ou discursifs. Les déplacements traduisent des engagements complexes et signifiants d'acteurs sociaux au sein de territoires : ils ne constituent pas de simples opérations de changement de position qu'une métrique pourrait décrire seulement en termes de quantité. Ce qui est engagé, 
lors d'un déplacement, ce sont des phénomènes comme les légitimités des acteurs concernés, les représentations qu'ils produisent du monde, de leurs territoires, de leurs institutions, de leurs identités et de leurs relations. Qu'il s'agisse d'un humain, d'un objet ou d'un animal, le déplacement traduit des phénomènes similaires, même si les termes de « légitimité » ou d'《 identité », ou encore celui de « territoire » sont connotés de manière différente. Cette manière d'envisager le territoire peut avoir des répercussions sur la notion sociologique de champ, en allant au delà de sa dimension quelque peu métaphorique : envisager les champs à la lumière des déplacements permet d'opérationnaliser cette notion en lui donnant une dimension matérielle ${ }^{16}$, puisqu'on peut qualifier - sans les épuiser pour autant - les relations qui s'instaurent entre acteurs sociaux en s'appuyant sur la description matérielle des déplacements qu'ils mettent en œuvre. En prenant le déplacement comme dimension d'analyse, on peut définir des indicateurs opératoires spécifiques à chaque terrain empirique. Cependant, les déplacements tels que nous les concevons demandent à l'enquêteur de se situer non dans l'analyse d'un champ clos sur lui-même, mais au niveau des frontières et des zones de communication entre différents champs et territoires. Quand les déplacements interviennent entre des champs professionnels, en particulier dans le contexte des institutions du savoir, les pôles reliés le sont par des organisations sociales et des dispositifs communicationnels qui prétendent à la transparence tandis qu'ils s'autonomisent: comme si chaque division structurelle ne pouvait que produire la médiation prétendant la résoudre. C'est aussi observable dans le cas du rapport à la nature: on ne célèbre jamais autant la nature pure et sauvage que quand le tourisme produit la « démocratisation » de son accès en développant des infrastructures. C'est en cela que le déplacement est producteur de sens, car il produit non seulement des discours, mais aussi de l'organisation et des dispositifs.

J'ai conscience de n'avoir fait qu'effleurer les problèmes théoriques et empiriques de la problématique du déplacement. II s'agissait avant tout de clarifier temporairement, en la soumettant à la discussion, une idée encore émergente qui demanderait encore de nombreuses investigations empiriques, et une érudition supérieure à la mienne, avant d'être correctement balisée. La figure de l'arpenteur médiéval pourrait servir d'emblème à cette tentative: utilisant parfois son corps - ses pieds, ses pas - comme unité de mesure, il agissait comme médiateur entre le pouvoir féodal, la paysannerie et les notaires des villes pour établir des divisions agraires ou urbaines. C'est par le contact avec le terrain, mais aussi avec les acteurs et témoins de la tradition orale du bornage. que l'arpenteur pouvait produire son expertise. II s'appuyait sur la légitimité de ses connaissances pratiques et géométriques, mais aussi sur son extériorité par rapports aux enjeux du territoire, ce qui garantissait son honnêteté. Une extériorité qui supposait cependant le contact avec les lieux, les gens et leur

16 P. Bourdieu (200 lb : 297) avait d'ailleurs pointé, brièvement, le lien entre les champs et les notions de distance et de déplacement. 
mémoire. La pratique de l'arpentage, et les instruments de sa mesure, étaient très hétérogènes et restent mal connue des historiens. Ces derniers font cependant I'hypothèse que les arpenteurs auraient contribué au passage à l'abstraction dans le rapport au territoire et à la progressive mathématisation de l'espace (Mousnier, 2004). Une fois cette rationalisation acquise, on peut faire comme si, du point de vue surplombant de la Raison, le corps n'aurait été qu'un obstacle à la connaissance. II semble pourtant en avoir été l'auxiliaire indispensable.

\section{Références}

Augoyard J.-F., 1979, Pas à pas. Essai sur les cheminements quotidiens en milieu urbain, Paris, Éd. Le Seuil.

Arnould P., Glon E., dirs, 2005, La nature a-t-elle encore une place dans les milieux géographiques?, Paris, Éd. Publications de la Sorbonne.

Auroux S., 2007, La question de l'origine des langues. Suivi de L'historicité des sciences, Paris, Presses universitaires de France.

Babou I., 1999, Science, télévision et rationalité. Analyse du discours télévisuel à propos du cerveau, Thèse en sciences de l'information et de la communication, Université Paris 7.

— 2004, Le cerveau vu par la télévision, Paris, Presses universitaires de France.

- 2009, Disposer de la nature. Enjeux environnementaux en Patagonie argentine, Paris, Éd. L'Harmattan.

— 2010, Rationalité \& nature. Une approche communicationnelle, Habilitation à diriger des recherches en sciences de l'information et de la communication, Université Paris 7.

Babou I., Le Marec J., 2003, « Science, musée et télévision : discours sur le cerveau », Communication et Langages, I38, pp. 69-88.

- 2009, « Les pratiques de communication professionnelle dans les institutions scientifiques: processus d'autonomisation », Revue d'anthropologie des connaissances, vol. 2, I, pp. II 5- I 42

Barbier R., Trépos J.-Y., 2007, « Humains et non-humains : un bilan d'étape de la sociologie des collectifs », Revue d'anthropologie des connaissances, vol. I, I, pp. 35-58.

Boas F., 1909, The Kwakiutl of Vancouver Island, New York, G. E. Stechert.

Boudes Ph., 2008, L'environnement, domaine sociologique. La sociologie française au risque de l'environnement, Thèse de doctorat en sociologie, Université Bordeaux 2.

Bourdieu P., 200 la, Science de la science et réflexivité, Paris, Éd. Raisons d'agir.

— $200 \mathrm{lb}$, Langage et pouvoir symbolique, Paris, Fayard.

Callon M., 1986, « Some elements of a sociology of translation : domestication of the scallops and the fishermen of St Brieuc Bay », pp. 196-223, in : Law J., dir., Power, action and belief: a new sociology of knowledge?, London, Routledge.

Collins H. M., Pinch T.J., 1979, «The Construction of the Paranormal : Nothing Unscientific is Happening », Wallis R., ed., Sociological Review Monograph, 27, pp. 237-270. 
Debaene V., 20 I0, L'adieu au voyage. L'ethnologie française entre science et littérature, Paris, Gallimard.

Descola P., 2002, L'anthropologie dans la nature, Paris, Éd. de l'École des hautes études en sciences sociales.

— 2005, Par-delà nature et culture, Paris, Gallimard.

Ethnologie française, 2009, « Les animaux de la discorde », Tome XXXIX.

Fouquier É., Véron E., 1985, Les spectacles scientifiques télévisés. Figures de la production et de la réception, Paris, Éd. La Documentation française.

Gorz A., 2008, Ecologica, Paris, Galilée.

Hert Ph., 2005, « Le terrain irréductible », Questions de communication, 8, pp. I 2 I - 34.

Jacob C., dir., 2007, Lieux de savoir. Espaces et communautés, Paris, A. Michel.

Juan S., 2006, Critique de la déraison évolutionniste. Animalisation de l'homme et processus de « civilisation », Paris, Éd. L'Harmattan.

- dir., 2007, Actions et enjeux spatiaux en matière d'environnement. De la contestation écologiste aux mesures de protection, Paris, Éd. L'Harmattan.

Latour B., 1985, « Les "vues" de l'esprit. Une introduction à l'anthropologie des sciences et des techniques », Culture technique, I4, pp. 4-30.

Le Marec J., 2002a, Ce que le «terrain » fait aux concepts : vers une théorie des composites, Habilitation à diriger des recherches en sciences de l'information et de la communication, Université Paris 7.

— 2002b, « Situations de communications dans la pratique de recherche : du terrain aux composites », Études de communication, 25, pp. I5-40.

Mattelart A., 1994, L'invention de la communication, Paris, Éd. La Découverte.

Merchet J.-D., 1999, « La vraie nature des géographes. Contre les écologistes, ils refusent de "mettre la nature sous cloche" et réhabilitent l'idée d"'exploitation" », Libération, 28 sept.

Moscovici S., 1972, La société contre nature, Paris, Union générale d'édition.

Mousnier M., 2004, « Mesurer les terres au Moyen-âge. Le cas de la France méridionale », Histoire et sociétés rurales, 22, 2e sem., pp. 29-63.

Olivesi S., 2007, « Le terrain. Une mythologie scientifique? », Questions de communication, 7. pp. $161-183$.

Passeron J.-Cl., 1995, « L'espace mental de l'enquête. La transformation de l'information sur le monde dans les sciences sociales », Enquête, I, pp. I3-42.

Petiteau J.-Y., 2008, « La méthode des itinéraires ou la mémoire involontaire », pp. I32154, in : Berque A., dir., Habiter dans sa poétique première, Paris, Éd. Donner Lieu.

Peirce Ch. S., 1978, Écrits sur le signe, trad. de l'anglais par G. Deledalle, Paris, Éd. Le Seuil.

Rastier Fr., 1990, « La triade sémiotique, le trivium et la sémantique linguistique », Nouveaux Actes sémiotiques, 9, pp. 5-40.

Roué M., 2009, « Une oie sauvage qui traverse les frontières. La bernache du Canada », Ethnologie française, I, Tome XXXIX, pp. 23-34. 
Rowntree V. J., McGuinness P., Marshall K. et al., 1998, « Increased harassment of right whales (Eubalaena Australis) by kelp gulls (Larus Dominicanus) at Península Valdés, Argentina », Marine Mammal Science, vol. I4, I, pp. 99- I I 5.

Rowntree V. J., Payne R. S., Schell D. M., 200I, « Changing patterns of habitat use by southern right whales (Eubalaena Australis) on their nursery ground at Península Valdés, Argentina, and in their long-range movements », Journal of Cetacean Research and Management, Special Issue, 2, pp. I33-144.

Saussure F. de, 1916, Cours de linguistique générale, Paris, Payot, 1996.

Selmi A., 2006, Administrer la nature, Paris, Éd. Quae.

Selmi A., Hirtzel V., dirs, 2007, Gouverner la nature, Paris, Éd. de L'Herne.

Servais V., 2005, « Enchanting and enchanted dolphins. An analysis of human/dolphin encounters 》, pp. 21 I-229, in : Knight J., ed., Animals in person, Oxford, Berg publisher.

Shapin S., 1991, « Une pompe de circonstance: la technologie littéraire de Boyle », pp. 37-86, in: Latour Br., Callon M., dirs, La science telle qu'elle se fait, Paris, Éd. La Découverte.

Thibaud J.-P., 1994, « Les mobilisations de l'auditeur-baladeur : une sociabilité publicative », Réseaux, vol. 12, 65, pp. 71-83.

— 2008, « Je, Tu, II. La marche aux trois personnes », Urbanism, 359, pp. 63-65.

Weber F., 1998, L'honneur des jardiniers. Les potagers dans la France du XXe siècle, Paris, Belin. 